\title{
Comparative Study of Hematological and Radiological Feature of Severe/Critically III Patients with COVID-19, Influenza A H7N9 and H1N1 Pneumonia
}

Jindan Kong

The First Affiliated Hospital of Soochow University

\section{Shan Wan}

Soochow University

\section{Sensen Shi}

The First Affiliated Hospital of Soochow University

\section{Songchao Cui}

The Second Affiliated Hospital of Soochow University

\section{Xiangping Zong}

The First Affiliated Hospital of Soochow University

\section{Jun Wang}

The first Affiliated Hospital of Soochow University

Jun Jin

The First Affiliated Hospital of Soochow University

\section{Mo Zhu}

The First Affiliated Hospital of Soochow University

\section{Di Zou}

The Affiliated Infectious Disease Hospital of Soochow University

\section{Depei Wu}

The First Affiliated Hospital of Soochow University

Jianhong Fu ( $\sim$ fujianhong@suda.edu.cn )

First Affiliated Hospital of Soochow University https://orcid.org/0000-0002-9044-024X

\section{Research article}

Keywords: COVID-19, Influenza A H7N9, H1N1, hematological, radiological

Posted Date: February 12th, 2021

DOI: https://doi.org/10.21203/rs.3.rs-228013/v1 
License: (c) (i) This work is licensed under a Creative Commons Attribution 4.0 International License. Read Full License

Version of Record: A version of this preprint was published at Journal of Clinical Laboratory Analysis on November 11th, 2021. See the published version at https://doi.org/10.1002/jcla.24100. 


\section{Abstract}

\section{Objectives}

This study aimed to explore useful clinical indexes for management of severe/critically ill patients with COVID-19, Influenza A H7N9 and H1N1 pneumonia by comparing hematological and radiological characteristics between them.

\section{Methods}

Severe/critically ill patients with confirmed diagnosis of COVID-19, Influenza A H7N9 and H1N1 pneumonia were retrospectively enrolled. The demographic data, clinical manifestations, hematological parameters, and radiological characteristics of three groups were compared. The influenza A was divided into two groups with/without patient death.

\section{Results}

In this study, 16 cases of COVID-19, 10 cases of influenza A (H7N9), and 13 cases of influenza A (H1N1) who met severe/critically ill criteria were included. Compared with COVID-19, the Influenza A (H7N9 and H1N1) groups had relatively more chronic diseases ( $80 \%$ and $92.3 \%$ vs $25 \%, \mathrm{P} \otimes 0.05)$, higher APACHE $\llbracket$ scores (16.00 \pm 8.63 and $15.08 \pm 6.24$, vs $5.50 \pm 2.58, P \unrhd 0.05)$ and higher mortality rates ( $40 \%$ and $46.2 \%$ vs $0 \%, P \otimes 0.05)$. The hematological finding indicated that Influenza A H7N9 and H1N1 patients had more significant lymphocytopenia $(0.59 \pm 0.31 \times 109 / \mathrm{L}$ and $0.56 \pm 0.35 \times 109 / \mathrm{L}$ vs $0.97 \pm 0.33 \times 109 / \mathrm{L}, \mathrm{P}<$ 0.05 ), elevated neutrophil to lymphocyte ratio (NLR; $14.67 \pm 6.10$ and $14.64 \pm 10.36$ vs $6.29 \pm 3.72$, $P<$ 0.05) compared to COVID-19. Especially in influenza A patients, NLR was significant different between the patients with or without death. Compared with the H7N9 group, ground glass opacity (GGO) on chest CT was more common in the COVID-19 group $(P=0.028)$, while pleural effusion was relatively rare $(P=$ $0.001)$.

\section{Conclusion}

Compared to COVID-19, patients with Influenza A (H7N9 and H1N1) had more underlying chronic diseases and higher mortality rates. The NLR can be used as a clinical parameter for the predication of risk stratification and outcome in COVID-19 and Influenza A pneumonia. Manifestations of pleural effusion or GGO in chest CT may be helpful for the identification of different viral pneumonia.

\section{Introduction}

In December 2019, a cluster pneumonia cases of unknown cause attacked Wuhan city in China. The pathogen was later identified to be a previously unknown beta coronavirus severe acute respiratory syndrome coronavirus 2 (SARS-CoV-2) [1]. The World Health Organization (WHO) denominated the new disease as coronavirus disease 2019 (COVID-19) in February and declared COVID-19 outbreak a pandemic in March, 2020. Until the October 1, 2020, more than 30 million confirmed COVID-19 cases 
have been reported globally, with more than 1,000,000 patient deaths. Till now there are no specific curative treatments or proven vaccines for COVID-19. Influenza A virus is another important type of contagious respiratory pathogen, which has caused several global epidemics in history. The Spanish flu in 1918 which caused tens of millions of deaths is mostly considered to be a virus closely related to Influenza A H1N1. Novel swine-origin influenza A (H1N1) virus identified in the United States in 2009 also caused a global pandemic [2]. In 2013, the novel avian-origin influenza A (H7N9) virus isolated in China had caused a sporadic epidemic, which was characterized by rapid progression and with a high fatality rate $[3,4]$.

The confirmed diagnosis of COVID-19, Influenza A pneumonia relies on reverse transcription-polymerase chain reaction (RT-PCR) from a nasopharyngeal swab, which need special laboratory and trained medical staffs. Hematological and radiological examinations are two basic methods for contagious viral pneumonias in clinical practice, which have the advantages of availability and short turnout time. In addition to RT-PCR testing, hematological and radiological examinations can be used for presumptive diagnosis. Although COVID-19 and Influenza A pneumonia are caused by two independent pathogens, there is still possibility that superimposed infection of influenza A and SARS-CoV-2 happens in the same patient [5]. Nevertheless, few study has reported the different clinical features between COVID-19, Influenza A H7N9 and H1N1 to date.

In this study, hematological and radiological characteristics of severe/critically ill patients with COVID-19, Influenza A (H7N9 and H1N1) pneumonias in Suzhou were analyzed. In this article, we aimed to find useful index for the management of COVID-19, Influenza A H7N9 and H1N1 patients.

\section{Materials And Methods}

\section{Patients}

This retrospective study was approved by the Institutional Ethics Committee of the first Affiliated Hospital of Soochow University. A total of 39 patients with severe/critically ill COVID-19, Influenza A H7N9 and H1N1 pneumonia diagnosed by RT-PCR in pharyngeal specimens in Suzhou center for disease control and prevention were included. The severe/critically ill COVID-19 patients were enrolled from January 10 to March 1, 2020. The severe/critically ill influenza A H7N9 and H1N1 patients were enrolled from April 03 to April 30 in 2013 and from November 27 to December 31 in 2009, respectively.

The epidemiological and clinical data, hematological and computed tomography (CT) results of included patients were collected through medical record system and recorded in a standard case questionnaire.

According to the Chinese guidelines for diagnosis and treatment of novel coronavirus infected pneumonia (trial version 6), patients who met one of the followings criterion were regarded as severe/critically ill cases: 1 ) respiratory rate $\geq 30 \mathrm{bpm} ; 2$ ) oxygen saturation $\leq 93 \% ; 3$ ) arterial partial pressure of oxygen (PaO2) / fraction of inspired oxygen ( $\mathrm{FiO2})<300 \mathrm{mmHg} ; 4)$ respiratory failure requires mechanical ventilation, shock or other organ failure, which requires ICU treatment. And 
severe/critically ill H7N9 and H1N1 pneumonia patients all met this criterion. Children were excluded. Two neutropenic patients with hematological malignancies after chemotherapy in H1N1 group were excluded.

\section{Laboratory Examination And Hospital Treatment}

The laboratory examination including complete blood count (CBC) and coagulation panel, were included for COVID-19, influenza A H7N9 and H1N1 patients. In the ICU, patients were managed with intensive care, which include antivirus, antibiotics, corticosteroids, fluid resuscitation, oxygen support and other affected vital organs support treatment after multidisciplinary discussions. Appropriate oxygen support methods, including nasal cannula (NC), high flow nasal cannula (HFNC), non-invasive ventilation (NIV), endotracheal intubation invasive mechanical ventilation (MV) and extracorporeal membrane oxygenation (ECMO) were applied according to the clinical condition.

\section{CT Imaging}

Patients' CT images were searched in the picture archiving and communication system (PACS). The first chest CT images after symptom onset were collected for analysis in different patients. Time (days) from the onset to CT scan were recorded at the same time. Manifestation of CT images in patients included ground-glass opacity (GGO), consolidation, distribution characteristics, and pleural effusion.

\section{Statistical analysis}

The statistical software SPSS 20.0 (IBM, Armonk, NY, USA) was used for data analysis. Continuous data with normal distribution were presented as mean \pm standard deviation and compared using One-way ANOVA, Kruskal-Wallis test and $t$-test. Categorical variables were presented as a percentage and assessed using $\chi 2$ test and Fisher's exact test. Two-sided $P \otimes 0.05$ indicated statistical significance.

\section{Results}

\section{Demographics}

A total of 16 cases of COVID-19, 10 cases of influenza A H7N9, and 13 cases of influenza A H1N1 were included. All of the patients met the aforementioned clinical classification criteria. The basic information was listed in Table 1. There was a difference in gender between $\mathrm{H} 7 \mathrm{~N} 9$ and $\mathrm{H} 1 \mathrm{~N} 1$ group $(P=0.029)$. The proportion of patients combined with underlying chronic diseases in H7N9 and H1N1 groups were higher than that in COVID-19 group ( $80 \%$ vs $25 \%, P=0.014$ and $92.3 \%$ vs $25 \%, P=0.000$ ). 
Table 1

Demographic and clinical characteristic of COVID-19, H7N9, H1N1 patients

\begin{tabular}{|c|c|c|c|c|}
\hline & COVID-19 & H7N9 & H1N1 & $\mathbf{P}$ \\
\hline & $N=16$ & $N=10$ & $N=13$ & \\
\hline Age (years) & $51.8 \pm 12.8$ & $62.7 \pm 17.8$ & $50.8 \pm 18.8$ & 0.177 \\
\hline Sex & & & & $0.042^{*}$ \\
\hline Male & $10(62.5 \%)$ & $9(90 \%)$ & $5(38.5 \%)$ & \\
\hline Female & $6(37.5 \%)$ & $1(10 \%)$ & $8(61.5 \%)$ & \\
\hline Chronic diseases & $4(25 \%)$ & $8(80 \%)$ & $12(92.3 \%)$ & 0.000 * \\
\hline Hypertension & $2(12.5 \%)$ & $5(50 \%)$ & $5(38.5 \%)$ & 0.110 \\
\hline Diabetes & $1(6.3 \%)$ & $4(40 \%)$ & $3(23.1 \%)$ & 0.109 \\
\hline Respiratory disease & $1(6.3 \%)$ & $2(20 \%)$ & $4(30.8 \%)$ & 0.214 \\
\hline Malignant tumor & $0(0 \%)$ & $1(10 \%)$ & $2(15.4 \%)$ & 0.334 \\
\hline Others & $2(12.5 \%)$ & $3(30 \%)$ & $4(30.8 \%)$ & 0.441 \\
\hline \multicolumn{5}{|l|}{ Symptoms } \\
\hline Fever & $15(93.8 \%)$ & $10(100 \%)$ & $13(100 \%)$ & 1.000 \\
\hline Cough & $10(62.5 \%)$ & $8(80 \%)$ & $13(100 \%)$ & $0.035^{\star}$ \\
\hline Expectoration & $7(43.8 \%)$ & $6(60 \%)$ & $10(76.9 \%)$ & 0.232 \\
\hline Chest tightness & $2(12.5 \%)$ & $6(60 \%)$ & $11(84.6 \%)$ & $0.000 *$ \\
\hline Days from onset to diagnosis (days) & $6.1 \pm 3.2$ & $8.3 \pm 4.6$ & $8.6 \pm 4.4$ & 0.208 \\
\hline APACHE II & $5.50 \pm 2.58$ & $16.00 \pm 8.63$ & $15.08 \pm 6.24$ & $0.000 *$ \\
\hline Death & $0(0 \%)$ & $4(40 \%)$ & $6(46.2 \%)$ & $0.003^{*}$ \\
\hline \multicolumn{5}{|l|}{ Treatment } \\
\hline Antivirus & $16(100 \%)$ & $10(100 \%)$ & $13(100 \%)$ & \\
\hline Antibiotics & $16(100 \%)$ & $10(100 \%)$ & $13(100 \%)$ & \\
\hline Corticosteroids & $16(100 \%)$ & $10(100 \%)$ & $13(100 \%)$ & \\
\hline Nasal cannula & $9(56.3 \%)$ & $2(20 \%)$ & $4(30.8 \%)$ & 0.163 \\
\hline High flow nasal cannula & $3(18.8 \%)$ & $0(0 \%)$ & $0(0 \%)$ & 0.106 \\
\hline
\end{tabular}

APACHE II Acute Physiology and Chronic Health Evaluation II, ECMO Extracorporeal Membrane Oxygenation, CRRT Continuous Renal Replacement Therapy, * $\mathrm{P}<0.05$. 


\begin{tabular}{|lllll|}
\hline & COVID-19 & H7N9 & H1N1 & P \\
\hline Noninvasive ventilation & $4(25 \%)$ & $1(10 \%)$ & $1(7.7 \%)$ & 0.531 \\
\hline Mechanical ventilation & $0(0 \%)$ & $7(70 \%)$ & $8(61.5 \%)$ & $0.000^{*}$ \\
\hline ECMO & $0(0 \%)$ & $2(20 \%)$ & $0(0 \%)$ & 0.061 \\
\hline Vasoactive drugs & $1(6.3 \%)$ & $5(50 \%)$ & $6(46.2 \%)$ & $0.016^{*}$ \\
\hline CRRT & $0(0 \%)$ & $4(40 \%)$ & $5(38.5 \%)$ & $0.008^{*}$ \\
\hline $\begin{array}{l}\text { APACHE II Acute Physiology and Chronic Health Evaluation II, ECMO Extracorporeal Membrane } \\
\text { Oxygenation, CRRT Continuous Renal Replacement Therapy, * } \mathrm{P}<0.05 .\end{array}$ & \\
\hline
\end{tabular}

The common symptoms in the three groups included fever, cough, expectoration and chest tightness. Cough was less common in the COVID-19 group than the H1N1 group ( $62.5 \%$ vs $100 \%, P=0.020)$. Compared to the COVID-19 group, chest tightness was more common in the H7N9 and H1N1 groups $(12.5 \%$ vs $60 \%, P=0.026$, and $12.5 \%$ vs $84.6 \%, P=0.000$ ). All patients received antivirus, antibiotics and corticosteroid therapy routinely.

Besides, the H7N9 and H1N1 groups had higher Acute Physiology and Chronic Health Evaluation (APACHE)- $\square$ scores than the COVID-19 group $(16.00 \pm 8.63$ vs $5.50 \pm 2.58, P=0.001 ; 15.08 \pm 6.24$ vs $5.50 \pm$ 2.58, $P=0.000$ ). The proportion of mechanical ventilation in $\mathrm{H} 7 \mathrm{~N} 9$ and $\mathrm{H} 1 \mathrm{~N} 1$ groups were significantly higher compared to the COVID-19 group (70\% vs $0 \%, P=0.000 ; 61.5 \%$ vs $0 \%, P=0.000)$. The application of vasoactive drugs ( $50 \%$ vs $6.3 \%, P=0.018$, and $46.2 \%$ vs $6.3 \%, P=0.026$ ) and continuous renal replacement therapy (CRRT) ( $40 \%$ vs $0 \%, P=0.014$, and $38.5 \%$ vs $0 \%, P=0.011$ ) was more common in H7N9 and H1N1 groups than the COVID-19 group. Among 16 COVID-19 patients, no one died during the time of observation, while four patients in the H7N9 group $(n=10)$ and six patients in the H1N1 group ( $n$ $=13$ ) died during the same period. Above all, there was a difference in mortality between the three groups; the mortality rate in the COVID-19 group was significantly lower than the H7N9 group $(P=0.014)$, and the H1N1 group $(P=0.004)$.

\section{Hematological Examination}

The results of complete blood count and coagulation panel on admission of all patients were summarized in Table 2. Although lymphopenia was detectable in all three groups, it was more pronounced in the H7N9 $(P=0.008)$ and H1N1 $(P=0.002)$ groups. The NLR was higher in the H7N9 group (Fold change $=1.33, P=0.006$ ) and the H1N1 group (Fold change $=1.33, P=0.003$ ). However, monocyte count in the COVID-19 group was significantly higher than the other two groups (Fold change $=1.21$ and $1.79, P=0.000)$. In the coagulation panel, fibrinogen level was slightly elevated in the COVID-19 group (Fold change $=0.77, P=0.001$ ), which was not available in the H7N9 and H1N1 groups. 
Table 2

Laboratory examination and chest CT findings of COVID-19, H7N9, H1N1 patients

\begin{tabular}{|c|c|c|c|c|}
\hline & COVID-19 & H7N9 & $\mathrm{H} 1 \mathrm{~N} 1$ & $\mathbf{P}$ \\
\hline CBC & $N=16$ & $N=10$ & $N=13$ & \\
\hline Leukocyte (× 109/L) & $7.14 \pm 3.61$ & $9.03 \pm 5.36$ & $7.05 \pm 2.82$ & 0.414 \\
\hline Neutrophil $\left(\times 10^{9} / \mathrm{L}\right)$ & $5.63 \pm 3.50$ & $8.17 \pm 4.97$ & $6.21 \pm 2.81$ & 0.243 \\
\hline Lymphocyte $\left(\times 10^{9} / \mathrm{L}\right)$ & $0.97 \pm 0.33$ & $0.59 \pm 0.31$ & $0.56 \pm 0.35$ & 0.004 * \\
\hline Monocyte $\left(\times 10^{9} / \mathrm{L}\right)$ & $0.53 \pm 0.14$ & $0.24 \pm 0.23$ & $0.19 \pm 0.17$ & 0.000 * \\
\hline Hemoglobin (g/L) & $135.88 \pm 16.46$ & $133.70 \pm 19.98$ & $112.23 \pm 30.90$ & 0.128 \\
\hline Platelets $\left(\times 10^{9} / \mathrm{L}\right)$ & $191.69 \pm 59.30$ & $157.40 \pm 58.24$ & $152.08 \pm 82.62$ & 0.249 \\
\hline NLR & $6.29 \pm 3.72$ & $14.67 \pm 6.10$ & $14.64 \pm 10.36$ & 0.004 * \\
\hline \multicolumn{5}{|l|}{ Coagulation panel } \\
\hline PT (sec) & $12.07 \pm 0.81$ & $12.51 \pm 1.52$ & $11.48 \pm 6.69$ & 0.499 \\
\hline APTT (sec) & $24.64 \pm 3.32$ & $35.21 \pm 26.35$ & $32.71 \pm 21.09$ & 0.335 \\
\hline Fibrinogen (g/L) & $5.31 \pm 1.58$ & $2.78 \pm 1.51$ & $3.32 \pm 1.85$ & 0.001 * \\
\hline Chest CT finding & $N=16$ & $N=8$ & $N=7$ & \\
\hline Days from onset to CT (days) & $5.13 \pm 3.22$ & $14.13 \pm 10.01$ & $16.00 \pm 9.15$ & 0.002 * \\
\hline Distribution & & & & 0.484 \\
\hline Unilateral lung & $0(0 \%)$ & $1(12.5 \%)$ & $0(0 \%)$ & \\
\hline Bilateral lungs & $16(100 \%)$ & 7 (87.5\%) & $7(100 \%)$ & \\
\hline GGO & $16(100 \%)$ & $5(62.5 \%)$ & $5(71.4 \%)$ & 0.028 * \\
\hline Consolidation & $10(62.5 \%)$ & $6(75 \%)$ & $5(71.4 \%)$ & 0.884 \\
\hline Pleural effusion & $1(6.3 \%)$ & $6(75 \%)$ & 3 (42.9\%) & 0.001 * \\
\hline
\end{tabular}

In the H7N9 and H1N1 groups, patients were evaluated and regrouped according to their survivals. After a further statistical analysis of their hematological examination, it was found that NLR was significantly higher in the group with patient death, compared to the group without patient death (Fold change $=0.66$, $P=0.033$; Table 3 ). 
Table 3

Laboratory examination of patients in influenza survival group and death group

Influenza Survival group

Influenza Death group

$\mathbf{P}$

$\mathrm{N}=13 \quad \mathrm{~N}=10$

CBC

Leukocyte $\left(\times 10^{9} / \mathrm{L}\right)$

$7.07 \pm 2.72$

$8.99 \pm 5.44$

0.327

Neutrophil $\left(\times 10^{9} / \mathrm{L}\right)$

$6.17 \pm 2.54$

$8.23 \pm 5.14$

0.267

Lymphocyte $\left(\times 10^{9} / \mathrm{L}\right)$

$0.65 \pm 0.35$

$0.47 \pm 0.27$

0.182

Monocyte $\left(\times 10^{9} / \mathrm{L}\right)$

$0.21 \pm 0.19$

$0.22 \pm 0.21$

0.922

Hemoglobin ( $\mathrm{g} / \mathrm{L}$ )

$120.54 \pm 32.92$

$122.90 \pm 22.66$

0.848

Platelets $\left(\times 10^{9} / \mathrm{L}\right)$

$165.08 \pm 88.86$

$140.50 \pm 40.06$

0.427

NLR

$11.37 \pm 5.81$

$18.93 \pm 9.97$

$0.033^{*}$

\section{Coagulation panel}

PT (sec)

$10.40 \pm 4.88$

$13.92 \pm 4.80$

0.099

APTT (sec)

$32.81 \pm 26.88$

$35.08 \pm 18.03$

0.820

Fibrinogen $(\mathrm{g} / \mathrm{L})$

$2.89 \pm 1.89$

$3.34 \pm 1.47$

0.538

CBC complete blood count, NLR neutrophil-lymphocyte ratio, PT prothrombin time, APTT activated partial thromboplastin time, ${ }^{*} \mathrm{P}<0.05$.

\section{CT Image Findings}

Sixteen cases of COVID-19, 8 cases of H7N9, and 7 cases of H1N1 patients had received chest CT examinations. In most patients with severe/critically ill viral pneumonia, the lesions dispersed in bilateral lungs $(\mathrm{n}=30)$. Compared with H7N9 group, GGO was more common in the COVID-19 group $(P=0.028)$, while pleural effusion was relatively rare in the COVID-19 group $(P=0.001)$. From the onset of symptoms to the first CT examination, the days in the COVID-19 group was significantly shorter than the other two groups (Median: $5.13 \pm 3.22$ days vs $14.13 \pm 10.01$ and $16.00 \pm 9.15$ days, $P<0.05$ ).

\section{Discussion}

The SARS-CoV-2, influenza A H7N9 and H1N1 viruses all belong to the RNA viruses, which spread through the respiratory tract or close contact, and damage multiple organs including the lungs [6]. The RT-PCR is widely used for the diagnose of patients with SARS-CoV-2 infection. However, the quantification of viral genomes cannot be used to evaluate the severity of pneumonia when planning management for patients. 
Nevertheless, the routine examination of patients with pneumonia through the hematological and radiological methods help clinicians to assess risk and predict prognosis of patients, which show special advantage when treating the patients with uncleared etiology. In this article, the clinical parameters of hematological and radiological examinations were comprehensively compared in between COVID-19 and $\mathrm{H} 7 \mathrm{~N}$ /H1N1 pneumonia, which indicate the different clinical characteristics of the three pneumonia.

In this research, we found the higher proportion of male patients in the H7N9 group, which was similar to the data from some previous clinical studies, which can be explained by more involvement of males in poultry breeding and slaughtering [7-9]. By comparison, cough and chest tightness were more common in the influenza groups compared with the COVID-19 group. However, these symptoms belong to the common symptoms of pneumonia, which are unspecific parameters useless for the distinguishing of different viral pneumonia. Therefore, careful inquiries of epidemiological history and clinical manifestations may be helpful. Interestingly, our research showed that those symptoms of COVID-19 patients were milder and had better prognosis compared with the influenza groups. The patients with COVID-19 had less underlying chronic disease and lower APACHE II score (within 24 hours) compared with the influenza groups. For this reason, the patients in the COVID-19 group received less life support treatment, and consequently had lower mortality compared with the influenza group. Similar to our findings, a case-control study found that the preexisting comorbidities (hypertension excluded) were significantly associated with human infection with H7N9 [10]. Moreover, a Spanish study in H1N1 showed that independent factors, including hospital-acquired infection, APACHE II score, underlying hematological diseases, continuous veno-venous hemofiltration, and mechanical ventilation were associated with higher mortality [11]. The COVID-19 group had milder symptoms and better survivals, which can be explained by the early diagnosis of COVID-19 due to the rapid response of medical system facing the urgent event [12]. Another explanation for this is the development of molecular medicine and emergency medicine from the prevalence year of H1N1 pneumonia (2009) to the outbreak of COVID-19 (2020). COVID-19 patients received more advanced testing method of RT-PCR, as well as better intensive care for critically ill patients, which accounts for the much better prognosis of COVID-19 compared to H1N1 or H7N9 patients.

Previous observations showed that severe influenza A H1N1 infections had marked lymphopenia detected by hematological examination $[13,14]$. Another case report detected more lymphocyte infiltration in the lung tissue from an autopsy of H1N1 patients [15]. Clinical studies also reported marked lymphopenia in patients with H7N9 infection, among which two death cases showed diffuse alveolar injury with lymphocyte and monocyte infiltration in percutaneous lung biopsies [7-9]. In the case of COVID-19, it was reported that hospitalized patients had some extent of lymphopenia, which was even more obvious in the patients with disease progression $[16,17]$. In our study, the lymphopenia was less obvious in the COVID-19 patients compared with the influenza groups, which is in consistent with the observations in other studies. A systematic review suggested that peripheral blood leucocyte ratio was an useful infection parameter for the distinguish of bacterial and viral infection [18]. Given the similar hemogram of patients with viral infections, a higher NLR was detected in the two influenza groups, especially in the influenza groups with patient death. A multi-center retrospective study also reported the 
NLR was an independent risk factor for patient survivals in H7N9 pneumonia [4]. A retrospective observational study found that the NLR was an easily measurable, available, cost-effective and reliable parameter, which continuous monitoring may be useful for the diagnosis and treatment of COVID-19 [19]. Taken together, these results reveal that the NLR in hematological examination is an important clinical parameter for the prediction of patient prognosis in the pneumonia caused by viral infections, including the influenza and COVID-19.

A single-center retrospective study of 242 COVID-19 cases with 52 patient death, found that the median absolute monocyte count was significantly reduced in the death group, while the NLR was significantly increased in the survival group [20]. In our research, there was no patient death in the COVID-19 group, with the median monocyte count within the normal range. One study conducted multivariate logistic regression analysis demonstrated that age, lymphocyte percentage and monocyte count were nonspecific laboratory markers predictive for COVID-19 [21]. As a result, the relevance of monocytes in prediction of severity of COVID-19 demands further research.

Studies had reported that COVID-19 patients had increased D-dimer and fibrin/fibrinogen degradation products, while abnormalities in prothrombin time, partial thromboplastin time, and platelet counts were relatively rare $[22,23]$. Three to four-fold increase of D-dimer levels was detected in the early stages of COVID-19 patients, which was associated with poor prognosis of COVID-19 patients [23]. Measuring the level of D-dimer and coagulation parameters from the early stage can also be useful in controlling and managing of COVID-19 disease [23]. Our data also showed that fibrinogen was elevated in the COVID-19 group, which suggested an activation of coagulation in the patients. Due to the lack of D-dimer data in the influenza groups, it was impossible to further compare the levels of D-dimer between the COVID-19 and the influenza groups.

A retrospective study of 1014 patients, which compared the accuracy between chest CT and RT-PCR in the diagnosis of potential COVID-19 patients, reported $59 \%$ positivity in RT-PCR and $88 \%$ positivity in chest CT, in which the chest CT had $97 \%$ accuracy in reference to the results of RT-PCR, which demonstrated the value of chest CT in diagnosis and monitoring the injury of COVID-19 [24]. Studies comparing the different manifestations of CT imaging between different viral pneumonia showed that the COVID-19 had areas of rounded opacity and septal thickening in peripheral regions of lungs, while the influenza A showed diffused distribution of lesions, including multiple nodules and "tree-in-bud" sign [25]. Another study compared the CT feature of $\mathrm{H} 7 \mathrm{~N} 9$ and $\mathrm{H} 1 \mathrm{~N} 1$ patients with acute respiratory distress syndrome (ARDS), which showed common manifestations, such as consolidation, GGO, air bronchogram, interlobular septal thickening, and nodular shadow, while pleural effusion was more specific in H7N9 pneumonia [26]. Compared to those studies, our study showed more GGO in the COVID-19 group, while pleural effusion was rare in COVID-19 group, but more common in the H7N9 group. The days from the onset to first CT examination was shorter in the COVID-19 group, due to the active response strategy and screening of patients with fever or respiratory symptoms in COVID-19, which led to the early diagnosis of COVID-19 with milder injury in the lungs. 
The limitations of this study are the small number of included cases, the long time span, the lack of comparison of the characteristics in mild cases, and the lack of inflammatory factors and infection markers.

\section{Conclusion}

In summary, our study revealed that patients with H7N9 and H1N1 had a more critical and complex condition, which led to more frequent life support treatment and a higher mortality rate in the ICU. Hematological examination showed slight decrease of lymphocyte, increase of monocyte, and slight increase of fibrinogen in the COVID-19 group compared with the influenza groups. The NLR in the influenza groups was significantly increased, especially in the subgroup with patient death. These results suggest that NLR can be used as an important clinical parameter for the assessment of severity of viral pneumonia. The manifestation in chest CT showed that pleural effusion and GGO may be helpful for the identification of COVID-19 from the influenza pneumonia.

\section{Declarations}

\section{Ethical approval and consent to participate}

Clinical data collection were approved by the Ethics Committee of the Soochow University (Suzhou, China).

\section{Consent for publication}

Not applicable

\section{Competing interests}

The authors declare no conflict of interests.

\section{Funding}

This study was supported by the National Natural Science Foundation of China (Grant No. 81730003), and partially supported by the Suzhou Science and Technology Bureau's Program (SS2019057).

\section{Authors' contributions}

$\mathrm{JK}, \mathrm{JF}$ and SW designed the work and performed patient data collection, analyzed the data, and wrote the manuscript. SS, SC, XZ, JW, JJ, MZ and DZ performed patient data collection in this retrospective clinical 
study. DW and JF revised the manuscript. All authors approved the submission.

\section{Acknowledgments}

The authors thank Professor Zhang Yukun, Jin Jun and Huang Xiaoping from the First Affiliated Hospital of Soochow University for their work in collecting patient data.

\section{Availability of data and materials}

The patient data analyzed in this study are not publicly available according to Chinese law restricting the sharing of non-anonymous patient data.

\section{References}

1. Zhu N, Zhang D, Wang W, Li X, Yang B, Song J, Zhao X, Huang B, Shi W, Lu R, et al. A Novel Coronavirus from Patients with Pneumonia in China, 2019. N Engl J Med. 2020;382(8):727-33.

2. Novel S-O, Influenza AVIT, Dawood FS, Jain S, Finelli L, Shaw MW, Lindstrom S, Garten RJ, Gubareva $\mathrm{LV}$, Xu X, Bridges CB, et al. Emergence of a novel swine-origin influenza A (H1N1) virus in humans. N Engl J Med. 2009;360(25):2605-15.

3. Gao R, Cao B, Hu Y, Feng Z, Wang D, Hu W, Chen J, Jie Z, Qiu H, Xu K, et al. Human infection with a novel avian-origin influenza A (H7N9) virus. N Engl J Med. 2013;368(20):1888-97.

4. Gong P, Xie Y, Jiang T, Liu Y, Wang M, Sun H, Zhang S, Zhang Y, Zhang X, Zhou J. Neutrophillymphocyte ratio predicts post-thrombolysis early neurological deterioration in acute ischemic stroke patients. Brain Behav. 2019;9(10):e01426.

5. Wu X, Cai Y, Huang X, Yu X, Zhao L, Wang F, Li Q, Gu S, Xu T, Li Y, et al. Co-infection with SARS-CoV-2 and Influenza A Virus in Patient with Pneumonia, China. Emerg Infect Dis. 2020;26(6):1324-6.

6. Hariri LP, North CM, Shih AR, Israel RA, Maley JH, Villalba JA, Vinarsky V, Rubin J, Okin DA, Sclafani A, et al: Lung Histopathology in Coronavirus Disease 2019 as Compared With Severe Acute Respiratory Sydrome and H1N1 Influenza: A Systematic Review. Chest 2020.

7. Gao HN, Lu HZ, Cao B, Du B, Shang H, Gan JH, Lu SH, Yang YD, Fang Q, Shen YZ, et al. Clinical findings in 111 cases of influenza A (H7N9) virus infection. N Engl J Med. 2013;368(24):2277-85.

8. Wan DM, Kang XH, Bai W, Zeng ZG, Zhang W. [The clinical characteristics and prognosis of 20 patients with H7N9 avian influenza]. Zhonghua Jie He He Hu Xi Za Zhi. 2019;42(10):750-4.

9. Yu WQ, Ding MD, Dai GH, Gu CJ, Xue L, Chen Y, Zhou DM, Xian JC, Xu HT. [Analysis of 15 cases of avian influenza virus (H7N9) infection]. Zhonghua Jie He He Hu Xi Za Zhi. 2018;41(7):534-8.

10. Ai J, Huang Y, Xu K, Ren D, Qi X, Ji H, Ge A, Dai Q, Li J, Bao C, et al: Case-control study of risk factors for human infection with influenza A(H7N9) virus in Jiangsu Province, China, 2013. Euro Surveill 2013, 18(26):20510. 
11. Alvarez-Lerma F, Marin-Corral J, Vila C, Masclans JR, Loeches IM, Barbadillo S, Gonzalez de Molina FJ, Rodriguez A, Group HNGSS. Characteristics of patients with hospital-acquired influenza A (H1N1)pdm09 virus admitted to the intensive care unit. J Hosp Infect. 2017;95(2):200-6.

12. Pan A, Liu L, Wang C, Guo H, Hao X, Wang Q, Huang J, He N, Yu H, Lin X, et al. Association of Public Health Interventions With the Epidemiology of the COVID-19 Outbreak in Wuhan, China. JAMA. 2020;323(19):1915-23.

13. Perez-Padilla R, de la Rosa-Zamboni D, Ponce de Leon S, Hernandez M, Quinones-Falconi F, Bautista E, Ramirez-Venegas A, Rojas-Serrano J, Ormsby CE, Corrales A, et al. Pneumonia and respiratory failure from swine-origin influenza A (H1N1) in Mexico. N Engl J Med. 2009;361(7):680-9.

14. Cui W, Zhao H, Lu X, Wen Y, Zhou Y, Deng B, Wang Y, Wang W, Kang J, Liu P. Factors associated with death in hospitalized pneumonia patients with 2009 H1N1 influenza in Shenyang, China. BMC Infect Dis. 2010;10:145.

15. Denney L, Aitken C, Li CK, Wilson-Davies E, Kok WL, Clelland C, Rooney K, Young D, Dong T, McMichael AJ, et al. Reduction of natural killer but not effector CD8 T lymphocytes in three consecutive cases of severe/lethal H1N1/09 influenza A virus infection. PLoS One. 2010;5(5):e10675.

16. Guan WJ, Ni ZY, Hu Y, Liang WH, Ou CQ, He JX, Liu L, Shan H, Lei CL, Hui DSC, et al. Clinical Characteristics of Coronavirus Disease 2019 in China. N Engl J Med. 2020;382(18):1708-20.

17. Zheng Y, Zhang Y, Chi H, Chen S, Peng M, Luo L, Chen L, Li J, Shen B, Wang D. The hemocyte counts as a potential biomarker for predicting disease progression in COVID-19: a retrospective study. Clinical chemistry laboratory medicine. 2020;58(7):1106-15.

18. Russell CD, Parajuli A, Gale HJ, Bulteel NS, Schuetz P, de Jager CPC, Loonen AJM, Merekoulias GI, Baillie JK. The utility of peripheral blood leucocyte ratios as biomarkers in infectious diseases: A systematic review and meta-analysis. J Infect. 2019;78(5):339-48.

19. Jimeno S, Ventura PS, Castellano JM, Garcia-Adasme SI, Miranda M, Touza P, Lllana I, LopezEscobar A. Prognostic implications of neutrophil-lymphocyte ratio in COVID-19. Eur J Clin Invest 2020:e13404.

20. Pakos IS, Lo KB, Salacup G, Pelayo J, Bhargav R, Peterson E, Gul F, DeJoy R 3rd, Albano J, PatarroyoAponte $\mathrm{G}$, et al. Characteristics of peripheral blood differential counts in hospitalized patients with COVID-19. Eur J Haematol. 2020;105(6):773-8.

21. Wang L, Liu Y, Zhang T, Jiang Y, Yang S, Xu Y, Song R, Song $M$, Wang L, Zhang W, et al. Differentiating Between 2019 Novel Coronavirus Pneumonia and Influenza Using a Nonspecific Laboratory Marker-Based Dynamic Nomogram. Open Forum Infect Dis. 2020;7(5):ofaa169.

22. Connors JM, Levy JH. COVID-19 and its implications for thrombosis and anticoagulation. Blood. 2020;135(23):2033-40.

23. Rostami M, Mansouritorghabeh H. D-dimer level in COVID-19 infection: a systematic review. Expert Rev Hematol. 2020;13(11):1265-75. 
24. Ai T, Yang Z, Hou H, Zhan C, Chen C, Lv W, Tao Q, Sun Z, Xia L. Correlation of Chest CT and RT-PCR Testing for Coronavirus Disease 2019 (COVID-19) in China: A Report of 1014 Cases. Radiology. 2020;296(2):E32-40.

25. Liu M, Zeng W, Wen Y, Zheng Y, Lv F, Xiao K. COVID-19 pneumonia: CT findings of 122 patients and differentiation from influenza pneumonia. Eur Radiol. 2020;30(10):5463-9.

26. Li H, Weng H, Lan C, Zhang H, Wang X, Pan J, Chen L, Huang J. Comparison of patients with avian influenza $A(\mathrm{H} 7 \mathrm{~N} 9)$ and influenza $A(\mathrm{H} 1 \mathrm{~N} 1)$ complicated by acute respiratory distress syndrome. Medicine. 2018;97(12):e0194.

\section{Figures}
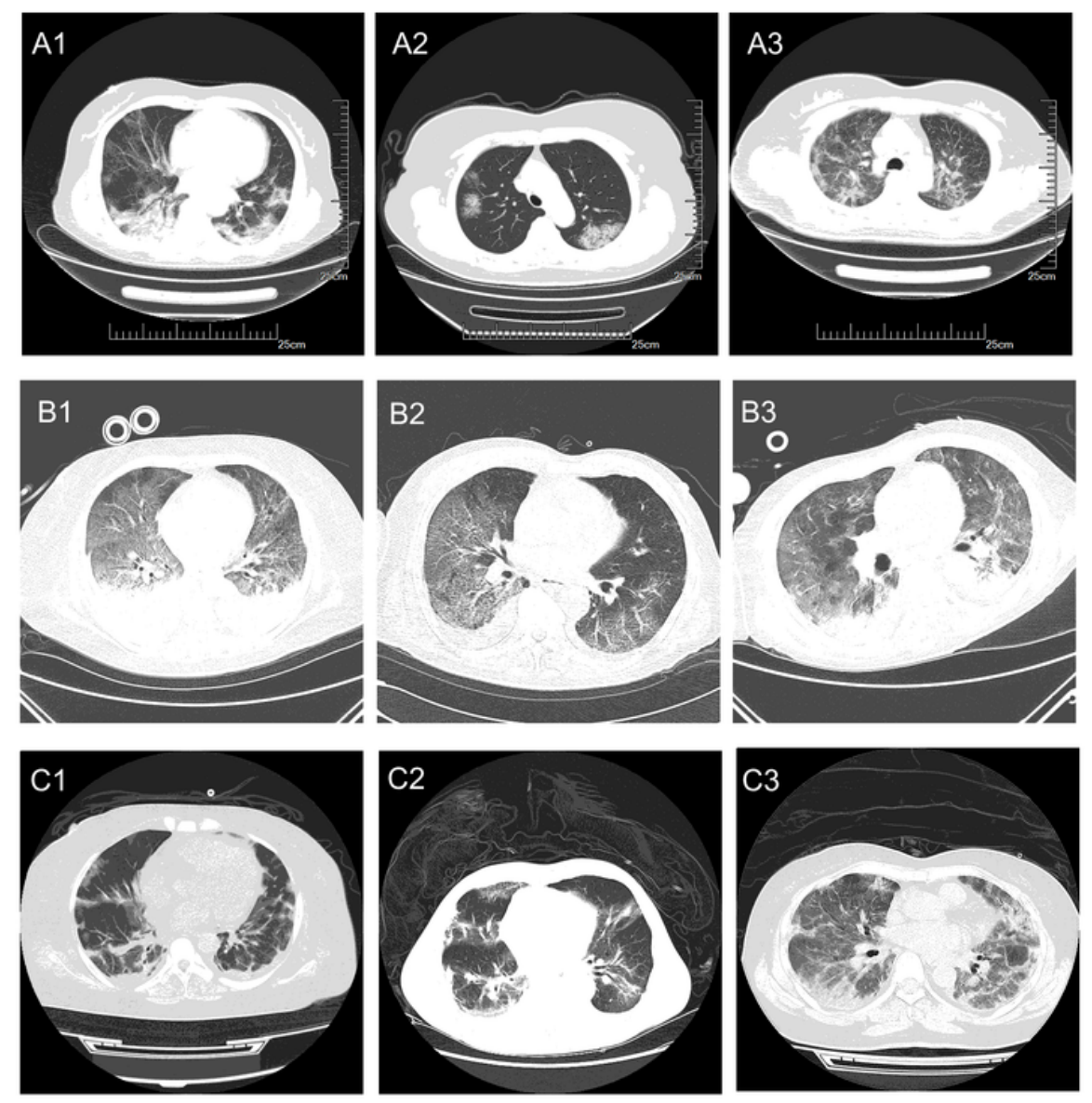

\section{Figure 1}

Representative chest CT images of severe/critically ill patients with COVID-19, influenza A H7N9 and H1N1 COVID-19: A1 (female/64), A2 (female/64), A3 (female/56). Chest CT showed multiple patchy and 
strip shadows in bilateral lung fields, more subpleural distribution, partially dense lesions. influenza $A$ H7N9: B1 (female/75), B2 (male/68), B3 (male/66). Chest CT showed diffuse patchy shadows of bilateral lungs with consolidation of lower lungs and pleural effusion. influenza A H1N1: C1 (female/40), C2 (male/36), C3 (female/49). Chest CT showed multiple patchy fuzzy shadows in bilateral lungs, with partial consolidation and pleural effusion.

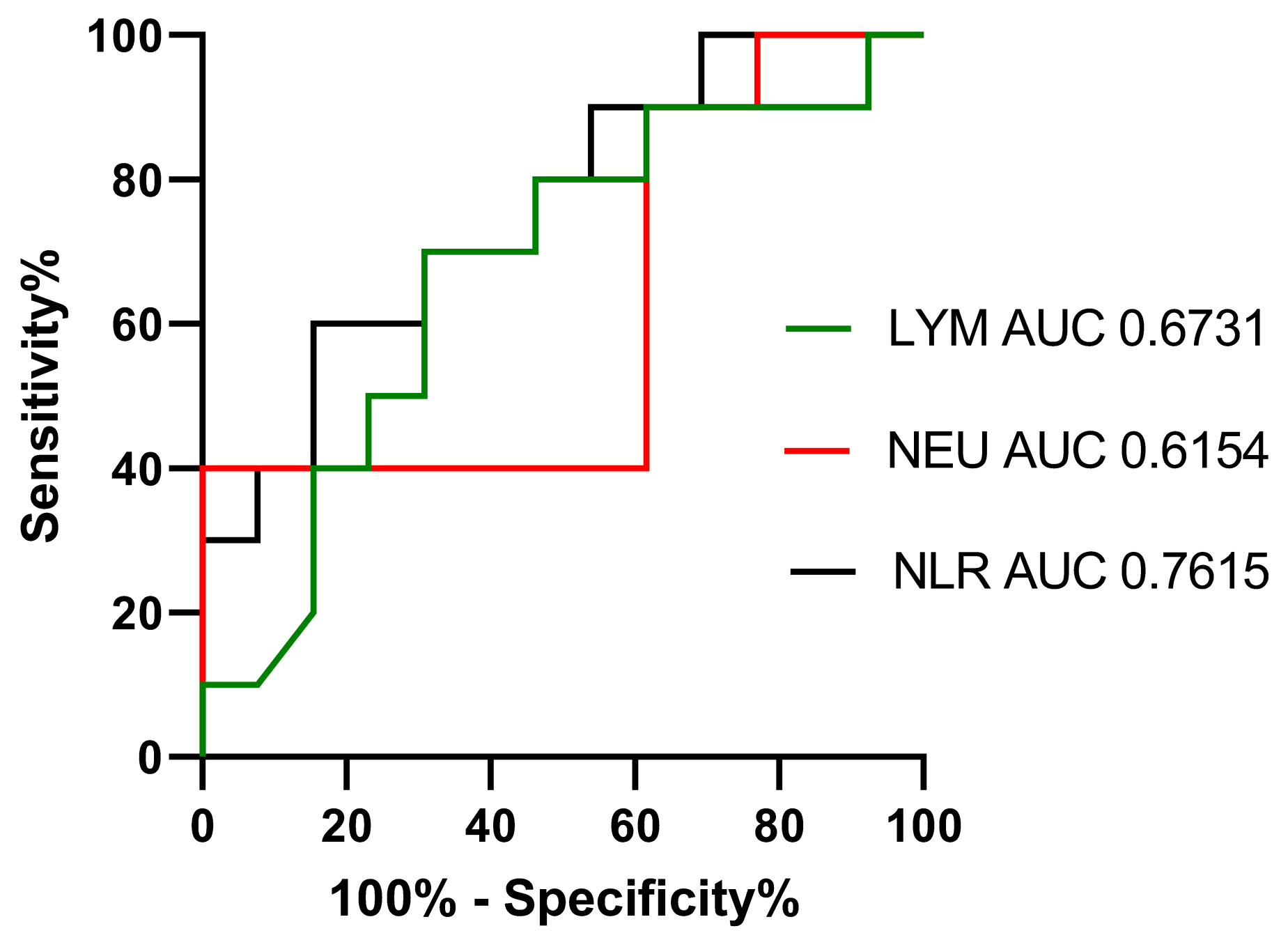

Figure 2

The receiver operating curve of lymphocyte, neutrophil and neutrophil to lymphocyte ratio (NLR) for death prediction of influenza A H7N9 and H1N1 